\title{
How do Clinicians View the Process of Shared Decision- Making with Parents Facing Extremely Early Deliveries? Results from an Online Survey
}

\author{
Brennan Hodgson Kim, MD ${ }^{1, *} \quad$ Jeanne Krick, MD, MA ${ }^{2, *} \quad$ Simone Schneider, MD ${ }^{3}$ Andres Montes, MD \\ Uchenna E. Anani, MD ${ }^{5}$ Peter D. Murray, MD, MSM ${ }^{6}$ Marin Arnolds, MD ${ }^{7}$ \\ Dalia M. Feltman, MD, $\mathrm{MA}^{8,90}$
}

${ }^{1}$ Department of Pediatrics, University of Chicago Comer Children's
Hospital, Chicago, Illinois
${ }^{2}$ Department of Pediatrics, San Antonio Military Medical Center, San
Antonio, Texas
${ }^{3}$ Department of Pediatrics, The University of North Carolina at Chapel
Hill, Chapel Hill, North Carolina
${ }^{4}$ Department of Obstetrics and Gynecology, St. Joseph's/Candler
Health System, Savannah, Georgia
${ }^{5}$ Department of Pediatrics, Vanderbilt University Medical Center,
Nashville, Tennessee
${ }^{6}$ Department of Pediatrics, University of Virginia School of Medicine,
Charlottesville, Virginia
${ }^{7}$ Department of Pediatrics, University of Michigan, Ann Arbor,
Michigan
${ }^{8}$ Evanston Hospital, NorthShore University HealthSystem, Evanston,
Illinois
${ }^{9}$ Pritzker School of Medicine, University of Chicago, Chicago, Illinois

Address for correspondence Dalia Feltman, MD, MA, Division of Neonatology, Department of Pediatrics, Evanston Hospital, NorthShore University HealthSystem, 2650 Ridge Avenue, Walgreen Building, Room 1505, Evanston, IL 60201

(e-mail:dfeltman@northshore.org;dmfeltman@gmail.com).

Am J Perinatol

\begin{abstract}
Objective The objective of this study was to better understand how neonatology (Neo) and maternal-fetal medicine (MFM) physicians approach the process of shared decision-making (SDM) with parents facing extremely premature ( $<25$ weeks estimated gestational age) delivery during antenatal counseling.

Study Design Attending physicians at U.S. centers with both Neo and MFM fellowships were invited to answer an original online survey about antenatal counseling for extremely early newborns. Preferences for conveying information are reported else-

Keywords

- antenatal counseling

- decision-making

- extreme prematurity

- maternal-fetal medicine

- neonatology

- ethical barriers

- resuscitation where. Here, we report clinicians' self-assessments of their ability to engage in deliberations and decision-making and perceptions of what is important to parents in the SDM process. Multivariable logistic regression analyzed respondents' views with respect to individual characteristics, such as specialty, gender, and years of clinical experience.

Results In total, 74 MFMs and 167 Neos representing $94 \%$ of the 81 centers surveyed responded. Neos versus MFMs reported repeat visits with parents less often $(<0.001)$ and agreed that parents were more likely to have made delivery room decisions before they counseled them less often $(p<0.001)$. Respondents reported regularly achieving
\end{abstract}

* These authors contributed equally to this work.

received

August 2, 2021

accepted after revision

December 9, 2021 (c) 2022. Thieme. All rights reserved.

Thieme Medical Publishers, Inc.,

333 Seventh Avenue, 18th Floor,

New York, NY 10001, USA
DOI https://doi.org/

10.1055/s-0041-1742186.

ISSN 0735-1631. 
most goals of SDM, with the exception of providing spiritual support. Most respondents reported that spiritual and religious views, risk to an infant's survival, and the infant's quality of life were important to parental decision-making, while a physician's own personal choice and family political views were reported as less important. While many barriers to SDM exist, respondents rated language barriers and family views that differ from those of a provider as the most difficult barriers to overcome.

Conclusion This study provides insights into how consultants from different specialties and demographic groups facilitate SDM, thereby informing future efforts for improving counseling and engaging in SDM with parents facing extremely early deliveries and supporting evidence-based training for these complex communication skills.

Newborns born between 22- and 25-week gestation are at a high risk of mortality, neurological impairment, and developmental delay due to their extreme prematurity. Given the uncertainty of the outcomes during this period, both obstetric and pediatric professional organizations support collaboration between clinicians and parents when making plans for delivery room care to determine what degree of resuscitation or comfort care to provide for the newborn. ${ }^{1,2}$ This collaborative process, referred to as shared decision-making (SDM), is defined as "an approach where clinicians and patients share the best available evidence when faced with the task of making decisions, [and] where patients are supported to consider options to achieve informed preferences." ${ }^{3}$ This mirrors the process described by adult critical care professional guidelines: "information exchange, deliberation, and making a treatment decision." ${ }^{4}$ The goal of SDM in this critical period is for parents and providers to work together to better understand the values that are most important to the family and to integrate them into delivery room care decisions for their infants. 5,6

While this practice of SDM bridges all disciplines of medicine, the perinatal period presents a particularly challenging setting. Many providers are poorly equipped to elicit and incorporate parental values into decisions for their extremely premature infants and often fall back on making decisions for infants themselves. ${ }^{7,8}$ Providers struggle to understand what information is most helpful to share with parents and the best methods for sharing it. ${ }^{6,9}$ Other elements that complicate discussions included a lack of clear data to provide parents, the dynamic nature of the peripartum period, the emotional qualities surrounding birth and newborns, and providers' own biases that may impact how medical choices and care options are presented. Interviews with physicians and parents have cited both institutional and ethical barriers as a contributing factor toward difficulty in practicing true SDM. Institutional barriers can include rotating medical teams, time limitations, and lack of training, while ethical barriers may include differing perspectives on both quality of life and how ethical principles apply to the care of extremely preterm newborns. $^{10-13}$

Both neonatal and obstetric providers interact with parents and are involved in making shared decisions with parents on behalf of their infants. While studies have previously demonstrated some differences in how these specialists provide antenatal counseling, the influence of other characteristics of individual providers on SDM is less clear. ${ }^{14,15}$ Improving counseling and teaching providers how to optimally engage parents in SDM require understanding current practices and attitudes of the three stakeholder groups (parents, maternal-fetal medicine [MFM], and neonatology [Neo]). We have drawn upon the rich body of literature examining these stakeholders' roles and practices and are studying elements of SDM for extremely early newborns with surveys of these three stakeholder groups. In a previous publication, we reported results of our survey of MFM and Neos that examined their prioritization of information given to parents in these antenatal counseling ahead of extremely early delivery. ${ }^{16}$ Here, we report from that survey these clinicians' views on the process of facilitating SDM for infants born at less than 25 weeks gestation, specifically, their perceived efficacy in achieving goals of communication, challenges to effective SDM, and analysis of individuals' SDM views with respect to their specialty and other characteristics. Clinicians' self-assessments of their strengths, weaknesses, and perceptions of what is important to parents can help focus educational efforts and will be compared with the responses of parents in an ongoing multicenter study of parents' preferences. In this way, we hope to contribute to evidence based on all three stakeholders to better guide clinicians engaging in this counseling.

\section{Materials and Methods}

\section{Questionnaire Development and Content}

To develop novel questions, an extensive literature review was conducted of publications regarding SDM. Articles were collected from PubMed by searching for the keywords "SDM + Neo," "SDM + obstetrics + prematurity," and "SDM + obstetrics + periviable." Results were sorted by "best match" and "recent first" and were further selected to include only articles examining at least in part the process of decisionmaking. One hundred and twenty-four articles were selected for review by investigators to identify critical SDM elements. Based on this literature review, survey questions were 
designed to elicit views on relevant SDM elements for antenatal consultation with parents anticipating extremely early delivery for which a decision on delivery room care would be shared. The survey consisted of three parts: views on facilitating the SDM process, respondents' extent of counseling practices and demographics, and information topics included in counseling (results of information topics are reported separately). ${ }^{16}$ Respondents were asked to reflect upon their own experiences of SDM counseling for parents facing extremely early delivery when both resuscitation and comfort care were being considered. Respondents rated on Likert scales their perceived efficacy in achieving goals of SDM supported by the literature (e.g., showing compassion, eliciting parents' hopes, and values), their perceptions of what parents find important to decision-making, the level of difficulty certain situations posed to SDM, and the appropriateness of sharing one's personal views as a clinician. Respondents reported individual characteristics including their gender identity, specialty, years of specialty experience, counseling frequency, and gestational ages at which they thought SDM for comfort care versus resuscitation was appropriate. The full survey can be found in this article's Supplemental Information.

\section{Survey Distribution}

Eight-hundred fifty-nine clinicians (289 MFMs and 570 Neos) were emailed invitations to participate in this online study in REDCap, a secure web-based application for managing online surveys. ${ }^{17}$ Clinicians were selected by emailing every third MFM or Neo attending physician listed on the institution Web site at every U.S. training center with both subspecialty fellowships. Email addresses were obtained from PubMed, Internet searches, and/or contacting their institutions. Ultimately, 11 individuals were removed from the eligibility pool after responding (dive no longer did clinical work or were retired, three had moved to different centers, and three were not actually neonatologists), changing the denominator to 848 (286 MFMs and 562 Neos). Responses were anonymous and voluntary, with incentives for charitable donations based on survey completion. This study was approved by the Institutional Review Board of NorthShore University Health System as exempt from requiring written informed consent under federal regulations. Respondents were informed in the introduction of the survey that their voluntary participation implied consent to analyze and share their anonymous responses with the academic community.

\section{Statistical Analysis}

Comparisons focused on differences between the responding MFM and Neo groups. Descriptive statistics were used to summarize responses to the questionnaires. Multivariable logistic regression was used to evaluate how survey responses varied with physicians' responses to questions of specialty, years of practice, gender, favoring SDM at each gestational age between 21 and 26 weeks, and whether they reported that parents have "usually" made a care decision prior to meeting the physician. Statistical significance was defined as a $p$-value $<0.05$. The statistical analysis was done using SigmaStat 4.0 (Systat Software, Inc., San Jose, CA).

\section{Results}

Two hundred and forty-one surveys were completed, with equal rates of participation by both specialties (74 of 286 MFMs, 25.9\% and 167 of 562 Neos, 29.7\%). Two invitees responded by declining, and two surveys were less than half complete, so removed, yielding an attrition rate of $0.5 \%$ (4 of 848). Despite low overall response rates, representation from each center was high. Of the 81 centers invited, only two centers had no respondents. Therefore, $94 \%$ ( 79 centers) were represented in our sample (54 centers, 67\% MFM programs and 74 centers, $91 \%$ of Neo programs).

\section{Respondent Characteristics and Clinical Practices by Specialty}

Grouped by specialty ( - Table 1), respondents were similar in their rates of gender, years of practice, and frequency of counseling encounters at this gestation. Regarding preferences for when to offer resuscitation, at 22 weeks, "usually" allowing parents a choice between resuscitation and comfort care was reported by $68 \%(n=114)$ of Neos and $54 \%(n=40)$ of MFMs, corresponding to an odds ratio of 1.8 (1.0-3.2, $p=0.05)$. Specialist groups were similar in their responses at all other weeks. MFM respondents reported seeing each individual patient with more continuity, most commonly reporting visits on a daily basis. No Neos saw parents daily before delivery; instead, most commonly reporting seeing patients as needed by medical team or parental request. Neos more often reported that the parents they counsel have already made a decision between resuscitation and comfort care before meeting with them.

\section{Achievement of Goals during SDM}

Reflecting on their antenatal counseling experiences for parents making delivery room care plans for their babies, respondents reported the frequency of achieving elements of SDM supported by current literature ( - Fig. 1). Respondents rated themselves least favorably in their achievement of providing spiritual support; in fact, this is the only goal reported as being achieved more infrequently than frequently (62 vs. 38\%). Eliciting family hopes, fears, and values was the second most infrequently achieved goal (14\%). Showing compassion was the goal reported most commonly as being achieved frequently or very frequently (28 and $71 \%$, respectively).

Multivariate analysis showed that several factors caused significant differences in rates of achieving goals of SDM selfreported as "very frequently." Responses showed significant differences when analyzed by multivariable regression for specialty, years of experience, gender, and when respondents believed parents had "usually" already decided prior to consultation. Half Neos versus MFMs were as likely to report being "very frequently" able to gauge parents' level of understanding and one-third as likely to report being able to "very frequently" provide spiritual support when 
Shared Decision-Making Process in Extremely Early Deliveries Kim et al.

\begin{tabular}{|c|c|c|c|c|c|}
\hline & \multicolumn{2}{|c|}{$\begin{array}{l}\text { MFM } \\
n=74\end{array}$} & \multicolumn{2}{|c|}{$\begin{array}{l}\text { Neo } \\
n=167\end{array}$} & \multirow[t]{2}{*}{ p-values } \\
\hline & $n$ & $\%$ & $n$ & $\%$ & \\
\hline \multicolumn{6}{|l|}{ Gender } \\
\hline Male & 26 & $35 \%$ & 70 & $42 \%$ & 0.61 \\
\hline Female & 47 & $64 \%$ & 95 & $57 \%$ & \\
\hline Other/No Answer & 1 & $1 \%$ & 2 & $1 \%$ & \\
\hline \multicolumn{6}{|l|}{ Years of practice } \\
\hline $0-7$ y & 27 & $36 \%$ & 50 & $30 \%$ & 0.60 \\
\hline $7-17$ y & 19 & $26 \%$ & 48 & $29 \%$ & \\
\hline$>17 \mathrm{y}$ & 28 & $38 \%$ & 69 & $41 \%$ & \\
\hline \multicolumn{6}{|c|}{ Frequency of consultations for threatened delivery 22-24 completed wk gestation } \\
\hline Once/twice each year & 2 & $3 \%$ & 14 & $8 \%$ & 0.10 \\
\hline Once/twice every few months & 21 & $28 \%$ & 59 & $35 \%$ & \\
\hline Once/twice each month & 33 & $45 \%$ & 62 & $37 \%$ & \\
\hline Once/twice each week & 15 & $20 \%$ & 31 & $19 \%$ & \\
\hline Once/twice each day & 3 & $4 \%$ & 1 & $1 \%$ & \\
\hline \multicolumn{6}{|c|}{ Usual number of antenatal meetings with a parent facing delivery 22 to 24 wk gestation } \\
\hline Once & 4 & $5 \%$ & 28 & $17 \%$ & $<0.001$ \\
\hline Routinely return one or more times & 17 & $23 \%$ & 15 & $9 \%$ & \\
\hline Return as needed per parent/team request & 22 & $30 \%$ & 121 & $72 \%$ & \\
\hline Weekly until delivery & 4 & $5 \%$ & 3 & $2 \%$ & \\
\hline Daily until delivery & 27 & $36 \%$ & 0 & $0 \%$ & \\
\hline \multicolumn{6}{|c|}{ How often do you think parents have already made their decision between resuscitation and comfort care before you meet them? } \\
\hline Rarely & 19 & $26 \%$ & 22 & $13 \%$ & $<0.001$ \\
\hline Sometimes & 38 & $51 \%$ & 57 & $34 \%$ & \\
\hline Often & 16 & $22 \%$ & 68 & $41 \%$ & \\
\hline Usually & 1 & $1 \%$ & 20 & $12 \%$ & \\
\hline
\end{tabular}

Abbreviations: MFM, maternal-fetal medicine; Neo, neonatology.

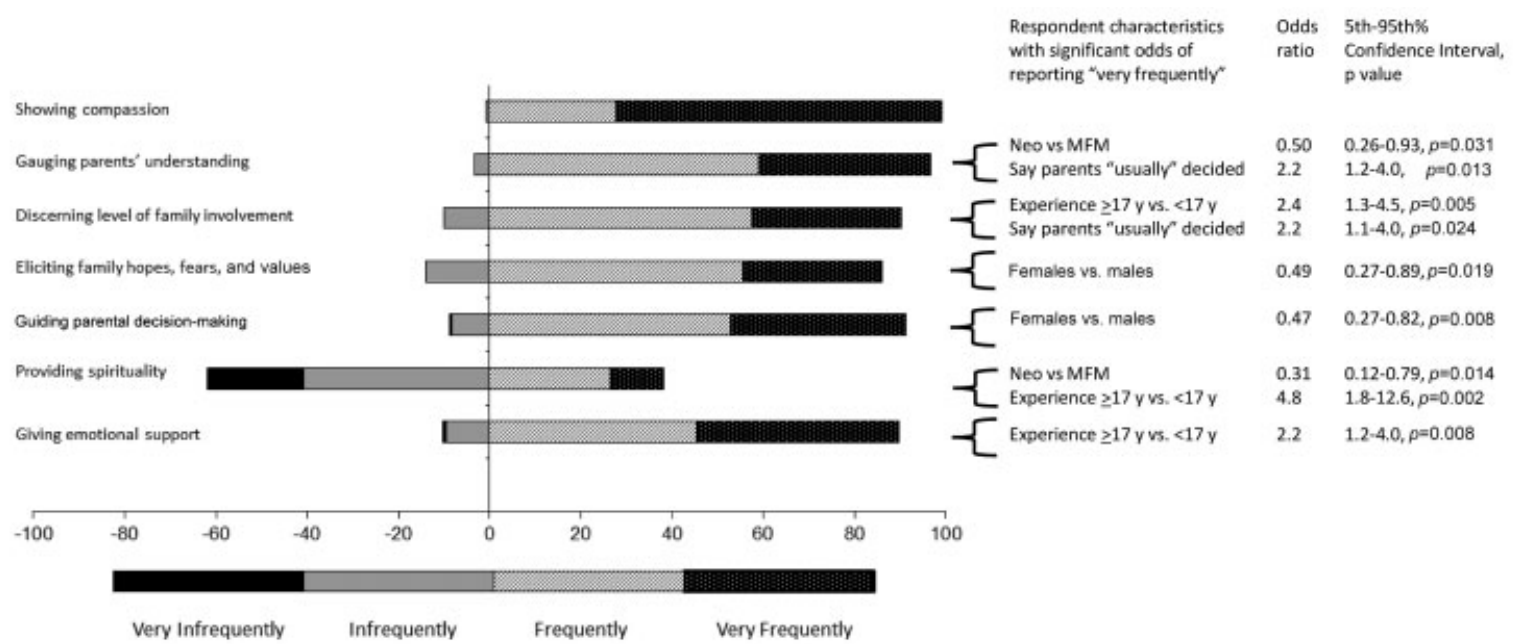

Fig. 1 Likert scales on facilitating shared decision-making. Achievement of specific goals when consulting (percent Likert scale responses). 
controlling for the other factors. Respondents with $\geq 17$ versus $<17$ years of experience in their field were more likely to report achieving several SDM goals "more frequently." More experienced physicians were more than twice as likely to report understanding how involved the family wanted to be in the SDM process, nearly five times as likely to provide spiritual support and more than twice as likely to provide emotional support. Female versus male respondents were about half as likely to report "very frequently" being able to elicit parents' hopes, fears, and values and help guide parents to a decision consistent with their values. Respondents who reported parents had "usually" already decided on comfort care versus resuscitation prior to consultation were more than twice as likely to report "very frequently" being able to gauge parents' level of understanding and understand how involved parents want to be in decisionmaking. The only category in which respondents did not differ based on provider characteristics was the rate of "very frequently" showing compassion/empathy, reported by $70 \%$ of Neos and 74\% of MFMs.

\section{Factors Clinicians Perceived as Important to Parents Making Decisions}

-Fig. 2 displays responses of physicians regarding how important certain factors seem to be to parents making decisions. The factors most often reported as commonly ("usually"|"nearly always") important to parents were spiritual/religious views (77\% MFMs and 80\% Neos) and risk of baby's survival ( $88 \%$ MFMs and $74 \%$ Neos), followed by quality of life of the child (76\% MFMs and 66\% Neos), and neurological development (72\% MFMs and 68\% Neos). Fewer than half (44\%) of respondents thought parents viewed family's quality of life as commonly important, and even fewer (15\%) thought sharing with parents what they them- selves would do in the case of their own child was commonly important.

There were no significant differences between responses based on specialty when rating how often certain factors were thought to be important to parents when deciding between comfort care and resuscitation. Multivariate analysis revealed that after correcting for other variables, respondents who felt parents had "usually" made a decision prior to consultation were only a third as likely to report neurological development as "nearly always important." While few respondents endorsed the influence of family's political views on decisions, when controlling for other factors, females versus males were four times more likely to report these were "nearly always important."

\section{Barriers to SDM}

Respondents were asked to rate the degree of difficulty different factors pose to facilitating SDM when they are present (-Fig. 3). The presence of language barriers was perceived most often as "very/extremely difficult" (51\%), followed by when physician's values differed from those of parents (48\%). When negotiated responsibility for decision making leaned more on physician input, $43 \%$ of respondents reported this being "very/extremely difficult," whereas when it leaned toward parental input, only $9 \%$ rated that level of difficulty. Some challenges, when present, tended to be reported as less difficult (only "moderately/minimally" as opposed to "very/extremely" difficult) in multivariable analysis for those with more clinical experience and for gender after controlling for the other factors of specialty and thinking parents usually have made a decision before consultation. After controlling for other factors, the more senior respondents (those with over 17 years' clinical experience) reported two to three times less difficulty when faced with

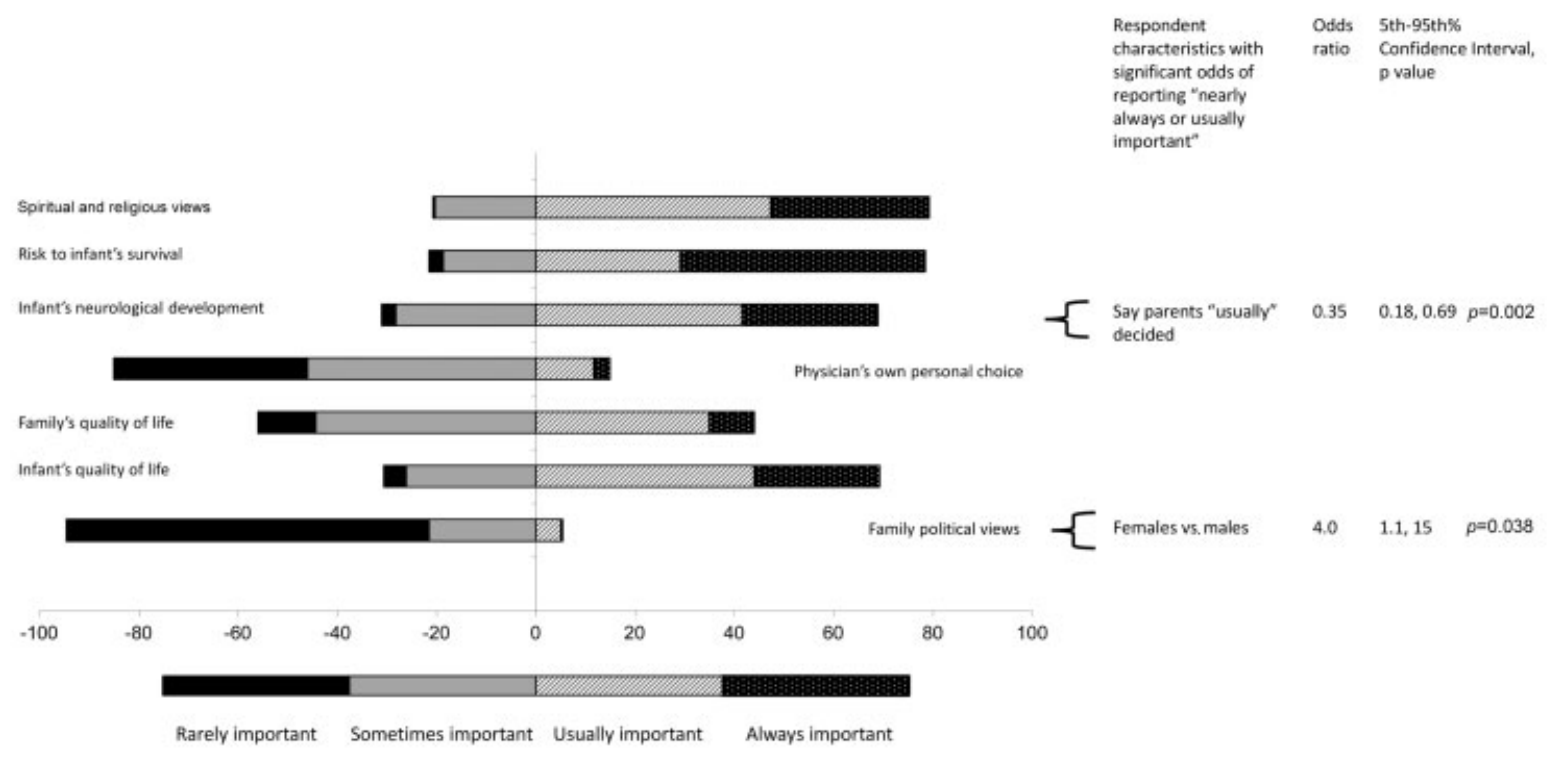

Fig. 2 Likert scales on facilitating shared decision-making. Importance of factors to parental decisions for resuscitation versus comfort care (percent Likert scale responses). 


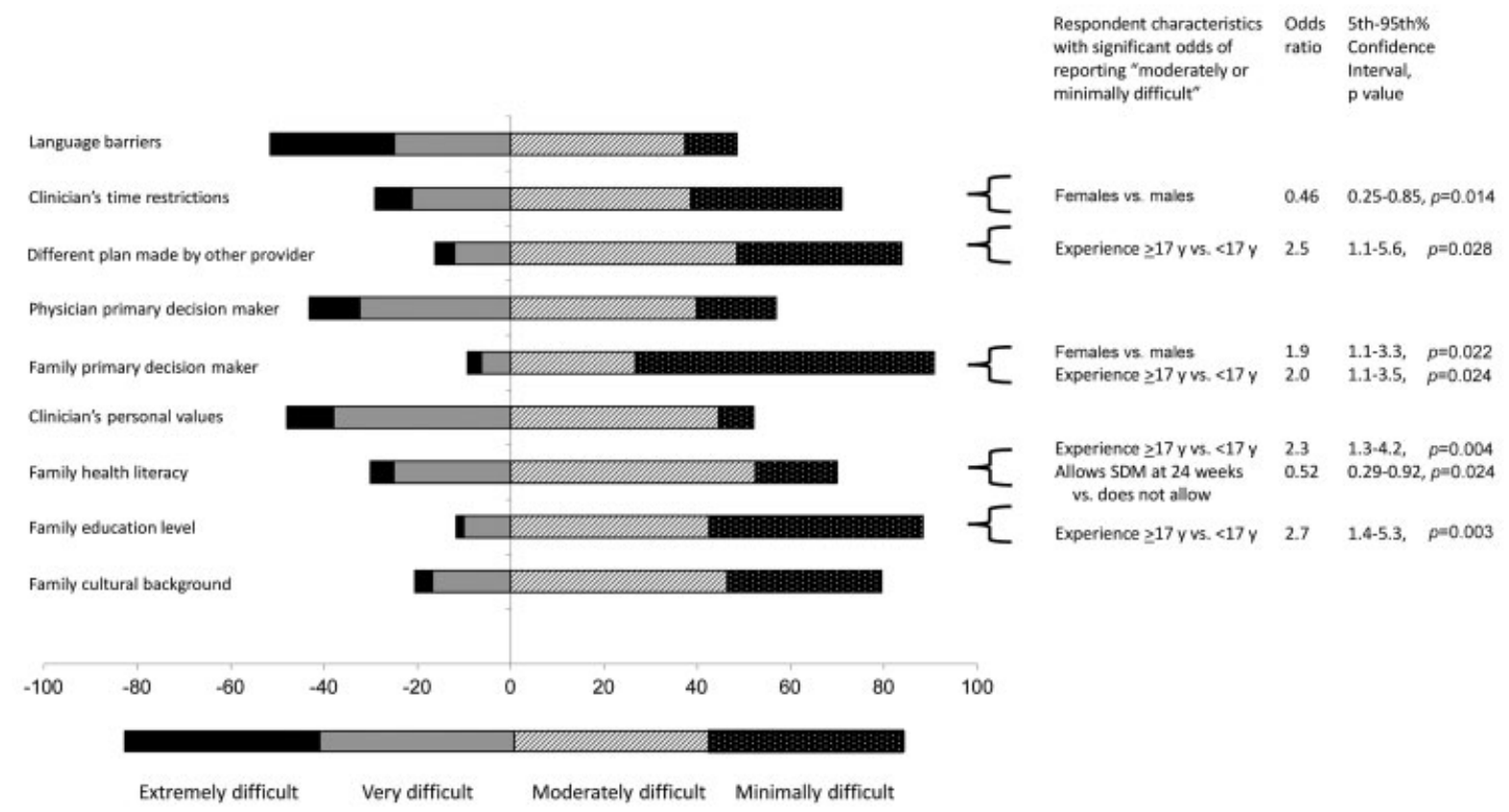

Fig. 3 Likert scales on facilitating shared decision-making. Difficulty of certain factors when present (percent Likert scale responses).

the following potential barriers to SDM: when responsibility for decision-making rested mostly on the clinician or mostly on the family and families' varying degrees of health literacy and education levels. Females reported decision-making responsibility resting mostly on the family was less difficult twice as often as male respondents, but females were about half as likely to report time constraints were less difficult compared with male colleagues.

\section{Sharing Personal Views}

More respondents reported sharing each of the personal views queried as inappropriate than appropriate on a Likert scale (-Fig. 4). When controlling for the other multivariable analysis factors, Neos versus MFMs were more than twice as likely to endorse the propriety of sharing what they would choose for their own child in this situation.

\section{Additional Variables Analyzed}

Respondents' specialty, gender, and years of clinical experience were most often found significant and, therefore, delineated by survey section. Less often, for a few survey questions, allowing choices for both resuscitation and comfort care at certain gestational weeks were significant. Respondents allowing SDM at 24 weeks were less likely to find different rates of health literacy problematic and more often endorsed sharing a physician's views of what they would do for their own child. Those allowing SDM at 21 weeks reported lower degrees of difficulty than others if decision-making responsibility rested more on the physician.

\section{Discussion}

To our knowledge, this is the first study to examine multiple facets of how clinicians view the process of SDM for extremely premature newborns rather than making decisions themselves. Knowing what stakeholders perceive as important, challenging, and appropriate will allow us to better study and teach techniques for this complicated physician skill set.

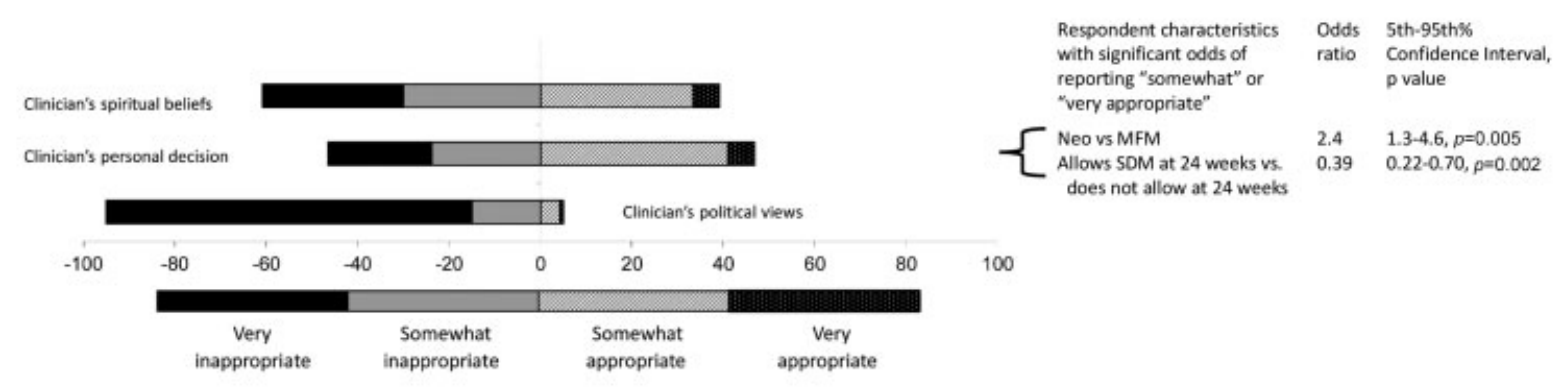

Fig. 4 Likert scales on facilitating shared decision-making. Appropriateness of sharing personal views as clinicians (percent Likert scale responses). 


\section{Current Gaps in SDM}

Parents frequently report religion and spirituality as being important in guiding their decision-making and coping with the stress of facing such an early delivery. ${ }^{18-22}$ Respondents recognized this, with $79 \%$ stating that spirituality is "usually" or "nearly always" important for parents. However, only $38 \%$ of physicians in this study stated that they "frequently" or "very frequently" provide spiritual support, notably lower than other metrics in the study. This gap between parents' needs and providers' skills is consistent with weakness in training programs, as reported in a survey of U.S. neonatology fellowship directors. ${ }^{23}$ A study of nearly 500 women at six U.S. centers revealed that only $9 \%$ of women had a chaplain consultation and only $20 \%$ of women had a social work consultation prior to delivery. ${ }^{24}$ If physicians are unable to provide this support themselves, it is important to ensure that parents are receiving this support from other sources, such as social workers or chaplains.

Prior studies have shown that both language and cultural differences between providers and parents represent a significant barrier to SDM. ${ }^{10,19}$ In this study, $12 \%$ of physicians reported cultural backgrounds being less of a barrier (moderately/minimally difficult) and 51\% reported language differences being less of a barrier. These data demonstrate that clinicians perceive a larger cultural barrier well beyond that produced by language. This could indicate ready access to translation services for language barriers but a lack of similar supports for cultural differences. The inclusion of cultural patient navigators could help with communication between these parties during SDM, as they could help parents address their needs in a socially appropriate and culturally competent way. ${ }^{25}$

\section{Barriers to SDM}

Physicians in this study felt that SDM was more challenging when the burden of decision-making was primarily on the clinician versus family (16\% moderately/minimally difficulty when burden on physician vs. $43 \%$ when burden on family). Parents have varying preferences for their involvement in decision-making, and some parents desire that clinicians make decisions for their infants. ${ }^{26-28}$ Research suggests that parents are better able to manage their distress following the loss of an infant if they felt they were involved in the decision but ultimately not solely responsible for making the final decision. ${ }^{29,30}$ Our results may reflect a general trend in Western medicine away from paternalistic decision-making, where physicians are much more comfortable deferring to parents to make decisions for their infants. This suggests moral comfort with administering either of the options given to parents, something built into the survey introductionthat clinicians should answer based on their counseling in situations in which resuscitation and comfort care are options. Our respondents reporting parents "often" or "usually" have made a decision prior to counseling were about twice as likely to report being able to gauge a family's understanding of the medical situation "often" or "usually" when controlling for other factors, perhaps reflecting again overall comfort with parents taking on more responsibility for a decision than the clinician. Whether physicians are accurately gauging understanding for parents who have or have not yet made decisions for their newborns requires further study.

\section{Comparing Specialties}

We previously reported that our survey respondent MFMs and Neos convey different information topics when counseling parents facing delivery at extremely early gestational ages. ${ }^{16}$ Here, we demonstrate that these specialists also differ in how they engage in the process of SDM with these families. Because they often are the primary practitioners for these hospitalized women, MFMs report seeing them with more continuity during their hospital stay. Perhaps not surprisingly, Neos reported greater frequencies of the parents they counsel having already made a decision before ever meeting with them which may have affected their subsequent responses on how they engage in facilitating these conversations. Neos were more likely than MFMs to endorse the propriety of discussing choices they would make for their own children with parents during SDM, contrary to another study reporting of obstetricians were more likely than neonatologists to make disclosures of personal choices for their own infants. ${ }^{14}$ Neos were, alternatively, less likely to report being able to gauge parents' level of understanding or to provide spiritual support to parents. These findings may reflect the unique aspects of each specialty, as Neos are likely more comfortable focusing their discussion on neonatal outcomes and often introduced as secondary consultants, whereas MFMs have frequently formed longitudinal relationships with parents. This could also reflect differences in training and background around SDM in the two specialties given the lack of standardized SDM approaches. While multiple factors likely contribute to specialists' differences, our findings reinforce the benefit of co-consultation.

\section{Comparing Respondents by Gender}

In this study, there were several differences between physicians self-identified as male and female in their approach to SDM for extremely premature infants. Female respondents noted less frequent achievement of two essential goals of SDM: eliciting parental values and assisting parents in making decisions concordant with these values. Additionally, women reported lower levels of difficulty in accomplishing SDM than their male counterparts when the balance of decision-making rested mostly on the family. Prior research has demonstrated that primary care female physicians tend to show more patient-centered communication in encounters but that this tendency is somewhat reversed in obstetric practitioners. $^{31,32}$ Given the self-reported nature of this study, it is also possible that differences in the ability to guide SDM may instead demonstrate a variance in confidence and perception rather than true differences in practice. Prior research has indicated that female physicians tend to report lower levels of confidence than their male counterparts, which may account for the differences seen in this study. $^{33,34}$ 
Female physicians also reported greater difficulty in communication due to time constraints. Past research has demonstrated that female primary care physicians spend an increased amount of time with patients when compared with their male counterparts. ${ }^{31}$ When coupled with this research, our findings may indicate that, when given similar amounts of time for counseling, female physicians may feel that they have less time to achieve similar goals leading to lower reported rates of achieving these features of SDM. Finally, female physicians reported that some values, including spirituality and political views, were more important for families during SDM than their male counterparts. Attempts to incorporate such values may contribute to perceived difficulties with time limitations while adequately guiding parents in making decisions.

\section{Comparing Years of Experience}

In this study, respondents with greater than 17 years of experience reported achieving SDM goals more often and had less difficulty with some potential barriers to SDM than their more junior counterparts. This supports prior research, which has shown that more experienced physicians are more likely to feel comfortable engaging parents in SDM and putting their values into action. ${ }^{8,35}$ In particular, they report being better able to provide spiritual and emotional support, aspects repeatedly shown in the literature to be important to parents engaging in SDM for infants born extremely premature. ${ }^{18-22}$ This difference may reflect the difficulty of learning to provide such support while maintaining professional boundaries, with the development of this skill coming only through years of experience and interactions with parents. Additionally, more experienced physicians reported less difficulty, overcoming certain barriers such as reduced health literacy and education. These scenarios require more customization to the family to ensure understanding and appropriate achievement of goals, skills which may also develop best through years of clinical experience. Even though every clinician develops and refines their communication skills throughout their career, methods of engaging trainee and junior physicians in both real and mock consultations to develop these critical skills require study. 23,36,37

\section{Limitations and Future Areas of Study}

Our survey results reflect clinicians' self-reported behaviors and perceptions of strengths and weaknesses rather than actual practices around SDM or the experiences of the parents they counsel. For example, language barriers may feel less problematic to clinicians than they would to parents. Future work should focus on the perceptions of parents during these encounters and how they relate to physicians' self-assessments. How elements like empathy and gauging understanding by the clinician contribute to a parent's overall trust and sense of partnership require further attention. Additionally, a selection bias may have caused some clinicians to choose to participate in our study. Nonetheless, views of physicians who counsel women facing extremely early delivery at nearly every U.S. center training MFM and Neo provide a solid foundation for the clinical study. Inves- tigations are planned to assess clinicians' use of these SDM elements and parents' views-the stakeholders missing from this study-on what is important to them in SDM.

\section{Conclusion}

Neonatologists and maternal-fetal medicine physicians guide parents through the difficult process of making choices for their infants that are born extremely premature through SDM. The results of this study reinforce the need for both specialties to engage in such counseling together, when possible, to provide robust and complementary support to these parents. Differences in how providers engage in this process based on their years of experience and gender may inform future efforts at improving skills related to SDM in specific provider groups. Finally, the study revealed current gaps in and barriers to SDM that must be addressed in future research and educational interventions to improve providers' ability to engage and support parents through this challenging process.

\section{Funding}

Data management was possible through grant support for REDCap from NorthShore University HealthSystem.

\section{Conflicts of Interest}

None declared.

\section{Acknowledgments}

Data management was possible through grant support for REDCap from NorthShore University HealthSystem. We thank Ms. Constance Herron BSN, MPH for her assistance in data collection for this study. We thank Ms. Sana Moqeet and Ms. Whitney Brown for their assistance in helping to compile contact information for our invited respondents.

\section{References}

1 Cummings JCOMMITTEE ON FETUS AND NEWBORN. Antenatal counseling regarding resuscitation and intensive care before 25 weeks of gestation. Pediatrics 2015;136(03):588-595

2 Obstetric Care Consensus No. 6 Periviable birth. Obstet Gynecol 2017;130(04):187-199

3 Elwyn G, Frosch D, Thomson R, et al. Shared decision making: a model for clinical practice. J Gen Intern Med 2012;27(10): 1361-1367

4 Kon AA, Davidson JE, Morrison W, Danis M, White DBAmerican College of Critical Care Medicine American Thoracic Society. Shared decision making in ICUs: an American College of Critical Care Medicine and American Thoracic Society Policy Statement. Crit Care Med 2016;44(01):188-201

5 Haward MF, Gaucher N, Payot A, Robson K, Janvier A. Personalized decision making: practical recommendations for antenatal counseling for fragile neonates. Clin Perinatol 2017;44(02): 429-445

6 Lantos JD. Ethical problems in decision making in the neonatal ICU. N Engl J Med 2018;379(19):1851-1860

7 Singh J, Fanaroff J, Andrews B, et al. Resuscitation in the "gray zone" of viability: determining physician preferences and predicting infant outcomes. Pediatrics 2007;120(03):519-526 
8 Bastek TK, Richardson DK, Zupancic JA, Burns JP. Prenatal consultation practices at the border of viability: a regional survey. Pediatrics 2005;116(02):407-413

9 Ruthford E, Ruthford M, Hudak ML. Parent-physician partnership at the edge of viability. Pediatrics 2017;139(04):e20163899

10 Barker C, Dunn S, Moore GP, Reszel J, Lemyre B, Daboval T. Shared decision making during antenatal counselling for anticipated extremely preterm birth. Paediatr Child Health 2019;24(04): 240-249

11 Shapiro MC, Donohue PK, Kudchadkar SR, Hutton N, Boss RD. Professional responsibility, consensus, and conflict: a survey of physician decisions for the chronically critically ill in neonatal and pediatric intensive care units. Pediatr Crit Care Med 2017;18(09): e415-e422

12 Ramsay SM, Santella RM. The definition of life: a survey of obstetricians and neonatologists in New York City hospitals regarding extremely premature births. Matern Child Health J 2011;15(04):446-452

13 Saigal S, Stoskopf BL, Feeny D, et al. Differences in preferences for neonatal outcomes among health care professionals, parents, and adolescents. JAMA 1999;281(21):1991-1997

14 Tucker Edmonds B, McKenzie F, Panoch JE, Wocial LD, Barnato AE, Frankel RM. "Doctor, what would you do?": physicians' responses to patient inquiries about periviable delivery Patient Educ Couns 2015;98(01):49-54

15 Tucker Edmonds B, McKenzie F, Panoch JE, Barnato AE, Frankel RM. Comparing obstetricians' and neonatologists' approaches to periviable counseling. J Perinatol 2015;35(05):344-348

16 Kim BH, Feltman DM, Schneider S, et al. What information do clinicians deem important for counseling parents facing extremely early deliveries?: results from an online survey Am J Perinatol 2021 Jun 7. doi: 10.1055/s-0041-1730430. Online ahead of print.

17 Harris PA, Taylor R, Thielke R, Payne J, Gonzalez N, Conde JG. Research electronic data capture (REDCap)-a metadata-driven methodology and workflow process for providing translational research informatics support. J Biomed Inform 2009;42(02):377-381

18 Boss RD, Hutton N, Sulpar LJ, West AM, Donohue PK. Values parents apply to decision-making regarding delivery room resuscitation for high-risk newborns. Pediatrics 2008;122(03):583-589

19 Drago MJ, Guillén U, Schiaratura M, et al. Constructing a culturally informed Spanish decision-aid to counsel Latino parents facing imminent extreme premature delivery. Matern Child Health J 2018;22(07):950-957

20 Keenan HT, Doron MW, Seyda BA. Comparison of mothers' and counselors' perceptions of predelivery counseling for extremely premature infants. Pediatrics 2005;116(01):104-111

21 Roscigno CI, Savage TA, Kavanaugh K, et al. Divergent views of hope influencing communications between parents and hospital providers. Qual Health Res 2012;22(09):1232-1246

22 Tucker Edmonds B, Savage TA, Kimura RE, et al. Prospective parents' perspectives on antenatal decision making for the antic- ipated birth of a periviable infant. J Matern Fetal Neonatal Med 2019;32(05):820-825

23 Feltman DM, Williams DD, Carter BS. How are neonatology fellows trained for antenatal periviability counseling? Am J Perinatol 2017;34(13):1279-1285

24 Feltman DM, Fritz KA, Datta A, et al. Antenatal periviability counseling and decision making: a retrospective examination by the investigating neonatal decisions for extremely early deliveries study group. Am J Perinatol 2020;37(02):184-195

25 Natale-Pereira A, Enard KR, Nevarez L, Jones LA. The role of patient navigators in eliminating health disparities. Cancer 2011;117(15, Suppl):3543-3552

26 Geurtzen R, van Heijst A, Draaisma J, et al. Prenatal counseling in extreme prematurity-insight into preferences from experienced parents. Patient Educ Couns 2019;102(08):1541-1549

27 Kavanaugh K, Savage T, Kilpatrick S, Kimura R, Hershberger P. Life support decisions for extremely premature infants: report of a pilot study. J Pediatr Nurs 2005;20(05):347-359

28 Moro TT, Kavanaugh K, Savage TA, Reyes MR, Kimura RE, Bhat R. Parent decision making for life support for extremely premature infants: from the prenatal through end-of-life period. J Perinat Neonatal Nurs 2011;25(01):52-60

29 Caeymaex L, Speranza M, Vasilescu C, et al. Living with a crucial decision: a qualitative study of parental narratives three years after the loss of their newborn in the NICU. PLoS One 2011;6(12): e28633

30 Caeymaex L, Jousselme C, Vasilescu C, et al. Perceived role in endof-life decision making in the NICU affects long-term parental grief response. Arch Dis Child Fetal Neonatal Ed 2013;98(01): F26-F31

31 Roter DLHJ, Hall JA, Aoki Y. Physician gender effects in medical communication: a meta-analytic review. JAMA 2002;288(06): 756-764

32 Roter DL, Hall JA. Physician gender and patient-centered communication: a critical review of empirical research. Annu Rev Public Health 2004;25:497-519

33 Gottlieb M, Chung A, Battaglioli N, Sebok-Syer SS, Kalantari A. Impostor syndrome among physicians and physicians in training: a scoping review. Med Educ 2020;54(02):116-124

34 Bakken LL, Sheridan J, Carnes M. Gender differences among physician-scientists in self-assessed abilities to perform clinical research. Acad Med 2003;78(12):1281-1286

35 Duffy D, Reynolds P. Babies born at the threshold of viability: attitudes of paediatric consultants and trainees in South East England. Acta Paediatr 2011;100(01):42-46

36 Arzuaga BH, Cummings CL. Practices and education surrounding anticipated periviable deliveries among neonatal-perinatal medicine and maternal-fetal medicine fellowship programs. J Perinatol 2016;36(09):699-703

37 Stokes TA, Watson KL, Boss RD. Teaching antenatal counseling skills to neonatal providers. Semin Perinatol 2014;38(01):47-51 